\title{
Axistationary perfect fluids - a tetrad approach
}

\author{
Gyula Fodor ${ }^{\S *}$ Mattias Marklund ${ }^{\uparrow \dagger}$ and Zoltán PerJés ${ }^{\S \ddagger}$ \\ $\S$ KFKI Research Institute for Particle and Nuclear Physics, \\ Budapest 114, P.O. Box 49, H-1525 Hungary \\ `Department of Plasma Physics, Umeå University, S-901 87 Umeå, Sweden
}

(September 4, 2018)

\begin{abstract}
Stationary axisymmetric perfect fluid space-times are investigated using the curvature description of geometries. Attention is focused on space-times with a vanishing electric part of the Weyl tensor. It is shown that the only incompressible axistationary magnetic perfect fluid is the interior Schwarzschild solution. The existence of a rigidly rotating perfect fluid, generalizing the interior Schwarzschild metric is proven. Theorems are stated on Petrov types and electric/magnetic Weyl tensors.
\end{abstract}

\section{Introduction}

A well-known property of the static, incompressible interior Schwarzschild space-time is conformal flatness, i.e., the Weyl tensor vanishing: $C_{i j k \ell}=0$. The Weyl tensor can be split into two parts, the electric and magnetic parts. The first part represents the tidal forces of the curvature, while the second part (which has no Newtonian analogue) is generated by the vorticity, shear, etc. of the congruence to which the electric and magnetic parts are related. Therefore, rotating matter can be viewed as partly responsible for the existence of the magnetic part of the curvature [1]. A simple example of a space-time containing rotating matter is expected to be a perfect fluid with a purely magnetic Weyl tensor.

Little is known about space-times with a magnetic curvature. Arianrhod et al. [2] have obtained an example of these space-times, albeit with an unknown equation of state. Another solution, suggested in [3] is, however, shown to be not purely magnetic by McIntosh [4].

To get some more insight on the subject, in this paper we investigate perfect fluid spacetimes with axistationary symmetry. We confine our attention to circularly rotating matter, i.e., the four-velocity lying in the plane of the two Killing vectors. Thus we may choose the line element in its Papapetrou form,

$$
\mathrm{d} s^{2}=f(\mathrm{~d} t+\omega \mathrm{d} \varphi)^{2}-f^{-1}\left[\mathrm{e}^{2 \lambda}\left(\mathrm{d} r^{2}+\mathrm{d} z^{2}\right)+\varrho^{2} \mathrm{~d} \varphi^{2}\right] .
$$

We choose the canonical frame

$$
\begin{array}{lll}
\boldsymbol{\theta}^{0} \equiv f^{1 / 2}(\mathrm{~d} t+\omega \mathrm{d} \varphi) & \boldsymbol{\theta}^{1} \equiv f^{-1 / 2} \mathrm{e}^{\lambda} \mathrm{d} r \\
\boldsymbol{\theta}^{2} \equiv f^{-1 / 2} \mathrm{e}^{\lambda} \mathrm{d} z & \boldsymbol{\theta}^{3} \equiv f^{-1 / 2} \varrho \mathrm{d} \varphi .
\end{array}
$$

and project all quantities onto this.

A space-time is locally completely determined by the set

$$
S \equiv\left\{R_{i j k \ell}, \gamma_{b k}^{a}, e_{i} x^{\alpha}, \eta_{i j}\right\}
$$

\footnotetext{
*E-mail: gfodor@rmki.kfki.hu

$\dagger$ E-mail: mattias.marklund@physics.umu.se

‡E-mail: perjes@rmki.kfki.hu
} 
where $R_{i j k \ell}$ are the components of the Riemann tensor, $\gamma_{b k}^{a}$ are the rotation coefficients, $\boldsymbol{e}_{i} x^{\alpha}$ the gradients of the essential coordinates, and $\eta_{i j}$ is the constant metric in the chosen frame $[5,6$. The set $S$ may be calculated using the frame (2) and has a form characteristic of the metric (1). We still have some freedom in performing Lorentz transformations of the frame (2), e.g., rotations in the (1,2)-plane (see Sec. 2). These transformations will change the appearance of the components of, say, the Riemann tensor, but will not introduce new non-zero components of this tensor. This freedom can be exploited to make the set $S$ as simple as possible. There is also a boost freedom, which does not introduce any new non-zero components of the tensors in the set $S$. This can be used to put the frame (2) in a comoving form, thus giving a natural interpretation of (some of) the Ricci rotation coefficients in terms of fluid variables (such as vorticity).

If we start with a set $S$, instead of generating the set $S$ from a metric satisfying Einstein's field equations, we need to impose certain restrictions on the elements of the set in order to guarantee that $S$ can be generated by a line-element that satisfy Cartan's equations under the restriction of Einstein's field equations. In general, these conditions are part of the commutator equations, Ricci equations, and Bianchi equations [5, 6].

The approach taken in this paper proceeds in the following steps:

1. Investigate the form of $S$ in the Lorentz frame (2) for the metric (11).

2. Analyze the properties of this $S$ under Lorentz transformations.

3. Using the knowledge obtained in the steps above, make an ansatz for the set $S$.

4. Subject the elements of this Ansatz to the necessary and sufficient equations (in general, parts of the commutator equations, Ricci equations, and Bianchi equations).

5. Use the fact that part of the variables in $S$ are the components of the Weyl tensor to investigate magnetic space-times.

In Sec. 2 we derive the equations describing an axisymmetric and stationary perfect fluid space-time. These are the commutators acting on the "essential coordinates" (on which all quantities in $S$ depend) and the Ricci equations. The Bianchi equations are not needed, but in practice they are very useful when there are restrictions on the Weyl tensor. All these equations are first order partial differential equations in terms of two variables for the quantities in the set $S$. Also, $S$ is chosen as to be compatible with the metric (11) in a Lorentz transformed version of the frame (2).

In Sec. 3 we investigate the restrictions of Sec. 2 in the case of a purely magnetic Weyl tensor. Specifically, we examine what space-times are allowed under the further restriction of incompressible fluid (constant energy density) or shear-free motion of the fluid (rigid rotation).

Section 1 describes how to obtain the full metric of the space-time. We focus on the physically important shear free space-times. We show that the line-element can be written in terms of quadratures once the elements of the set $S$ are determined by the equations set up in Sec. 2.

In Sec. 5, we consider various Petrov types of circularly rotating perfect fluids. We prove that type $\mathrm{N}$ fluids have the unphysical equation of state $\mu+p=0$, and that rigidly rotating purely electric fluids are of Petrov type D.

\footnotetext{
${ }^{1}$ Note that the analysis would be possible in any constant frame, not just the Lorentz frame. We choose this particular frame since it is related, in a simple fashion, to the physical observables of the fluid.
} 


\section{Equations}

Our space-time possesses two Killing vectors, one time-like and one space-like, which commute. We assume that the coordinates are adapted to the Killing vectors, such that all quantities in the set $S$ are dependent on two coordinates $\left\{x^{\alpha}\right\} \equiv\{x, y\}$.

The source is a perfect fluid with the energy-momentum tensor

$$
T_{i j}=(\mu+p) u_{i} u_{j}-p g_{i j} .
$$

We choose a frame $\boldsymbol{e}_{i} \equiv\left(\boldsymbol{e}_{i} x^{\mu}\right)\left(\partial / \partial x^{\mu}\right)$ with dual $\boldsymbol{\omega}^{i} \equiv \omega_{\mu}^{i} \mathrm{~d} x^{\mu}, i=0, \ldots, 3$, such that

$$
\begin{aligned}
& \mathrm{d} s^{2}=\left(\boldsymbol{\omega}^{0}\right)^{2}-\left(\boldsymbol{\omega}^{1}\right)^{2}-\left(\boldsymbol{\omega}^{2}\right)^{2}-\left(\boldsymbol{\omega}^{3}\right)^{2} \\
& \boldsymbol{e}_{0} \equiv \boldsymbol{u}
\end{aligned}
$$

i.e., the frame is comoving and $\left(g_{i j}\right)=\left(\eta_{i j}\right) \equiv \operatorname{diag}(1,-1,-1,-1)$. In terms of the frame (2) related to the metric (11), the choice of a comoving frame means that we have carried out a boost in the (0,3)-plane:

$$
\left(\begin{array}{l}
\boldsymbol{\theta}^{0} \\
\boldsymbol{\theta}^{1} \\
\boldsymbol{\theta}^{2} \\
\boldsymbol{\theta}^{3}
\end{array}\right) \mapsto\left(\begin{array}{cccc}
\cosh \chi & 0 & 0 & \sinh \chi \\
0 & 1 & 0 & 0 \\
0 & 0 & 1 & 0 \\
\sinh \chi & 0 & 0 & \cosh \chi
\end{array}\right)\left(\begin{array}{l}
\boldsymbol{\theta}^{0} \\
\boldsymbol{\theta}^{1} \\
\boldsymbol{\theta}^{2} \\
\boldsymbol{\theta}^{3}
\end{array}\right)
$$

or explicitly

$$
\begin{aligned}
\boldsymbol{\theta}^{0} \mapsto \boldsymbol{\theta}^{0} & =\cosh \chi f^{1 / 2}(\mathrm{~d} t+\omega \mathrm{d} \varphi)+\sinh \chi f^{-1 / 2} \varrho \mathrm{d} \varphi \\
\boldsymbol{\theta}^{3} \mapsto \boldsymbol{\theta}^{3} & =\cosh \chi f^{-1 / 2} \varrho \mathrm{d} \varphi+\sinh \chi f^{1 / 2}(\mathrm{~d} t+\omega \mathrm{d} \varphi),
\end{aligned}
$$

where $\chi=\chi(x, y)$. In the case of a rigidly rotating fluid, the boost parameter $\chi$ may always be put to zero. With this, the contravariant vectors $\boldsymbol{e}_{0}$ and $\boldsymbol{e}_{3}$ become

$$
\begin{aligned}
& \boldsymbol{e}_{0}=\frac{1}{\varrho}\left[\left(\sinh \chi f^{1 / 2} \omega+\cosh \chi f^{-1 / 2} \varrho\right) \partial_{t}-\sinh \chi f^{1 / 2} \partial_{\varphi}\right] \\
& \boldsymbol{e}_{3}=\frac{1}{\varrho}\left[-\left(\cosh \chi f^{1 / 2} \omega+\sinh \chi f^{-1 / 2} \varrho\right) \partial_{t}+\cosh \chi f^{1 / 2} \partial_{\varphi}\right] .
\end{aligned}
$$

We define the non-zero components of the Riemann tensor of the circularly rotating fluid in the frame given by Eqs. (5) and (6) as

$$
\begin{array}{ll}
R_{0101}=E_{1}-\frac{1}{6}(\mu+3 p) & R_{0102}=E_{3} \\
R_{0113}=-H_{3} & R_{0123}=H_{1} \\
R_{0202}=E_{2}-\frac{1}{6}(\mu+3 p) & R_{0213}=-H_{2} \\
R_{0223}=H_{3} & R_{0303}=-E_{1}-E_{2}-\frac{1}{6}(\mu+3 p) \\
R_{0312}=-H_{1}-H_{2} & R_{1212}=E_{1}+E_{2}-\frac{1}{3} \mu \\
R_{1313}=-E_{2}-\frac{1}{3} \mu & R_{1323}=E_{3} \\
R_{2323}=-E_{1}-\frac{1}{3} \mu, &
\end{array}
$$

where $E_{A}$ and $H_{A}, A=1,2,3$, are components of the electric part $E_{i j} \equiv C_{i k j \ell} u^{k} u^{\ell}$ and magnetic part $H_{i j} \equiv{ }^{*} C_{i k j \ell} u^{k} u^{\ell}$ of the Weyl tensor respectively. The rotation coefficients 
$\gamma_{j k}^{i} \equiv \omega_{\mu ; \nu}^{i}\left(\boldsymbol{e}_{j} x^{\mu}\right)\left(\boldsymbol{e}_{k} x^{\nu}\right)$ are

$$
\begin{array}{ll}
\gamma_{010}=-a_{1} & \gamma_{020}=-a_{2} \\
\gamma_{013}=-\sigma_{2}-\omega_{2} & \gamma_{023}=\sigma_{1}+\omega_{1} \\
\gamma_{031}=-\sigma_{2}+\omega_{2} & \gamma_{032}=\sigma_{1}-\omega_{1} \\
\gamma_{121} \equiv \alpha_{1} & \gamma_{122} \equiv-\alpha_{2} \\
\gamma_{130} \equiv \gamma_{2} & \gamma_{133} \equiv \beta_{1} \\
\gamma_{230} \equiv-\gamma_{1} & \gamma_{233} \equiv \beta_{2}
\end{array}
$$

where $a_{i}$ is the acceleration, $\sigma_{i}$ the shear, and $\omega_{i}$ the vorticity of the fluid.

We still have the freedom to perform rotations

$$
\left(\begin{array}{c}
\boldsymbol{\omega}^{0} \\
\boldsymbol{\omega}^{1} \\
\boldsymbol{\omega}^{2} \\
\boldsymbol{\omega}^{3}
\end{array}\right) \mapsto\left(\begin{array}{cccc}
1 & 0 & 0 & 0 \\
0 & \cos \vartheta & \sin \vartheta & 0 \\
0 & -\sin \vartheta & \cos \vartheta & 0 \\
0 & 0 & 0 & 1
\end{array}\right)\left(\begin{array}{l}
\boldsymbol{\omega}^{0} \\
\boldsymbol{\omega}^{1} \\
\boldsymbol{\omega}^{2} \\
\boldsymbol{\omega}^{3}
\end{array}\right)
$$

in the (1,2)-plane (here $\vartheta=\vartheta(x, y)$ ). 2 This rotation will not introduce any new non-zero components in the Riemann tensor or the rotation coefficients (as any other rotation would do), although the components of the respective geometric objects will mix.

Parts of the Ricci equations and Bianchi equations are solved by the choice $\boldsymbol{e}_{0} x=\boldsymbol{e}_{0} y=$ $\boldsymbol{e}_{3} x=\boldsymbol{e}_{3} y=0$. Using the reduced number of coordinate gradients, we may write

$$
\begin{aligned}
& \boldsymbol{e}_{1}=\xi_{1} \partial_{x}+v_{1} \partial_{y} \\
& \boldsymbol{e}_{2}=\xi_{2} \partial_{x}+v_{2} \partial_{y},
\end{aligned}
$$

where $\xi_{i} \equiv \boldsymbol{e}_{i} x, v_{i} \equiv \boldsymbol{e}_{i} y$.

The commutator equations are generated by

$$
\left[\boldsymbol{e}_{i}, \boldsymbol{e}_{j}\right]=\left(\gamma^{k}{ }_{j i}-\gamma^{k}{ }_{i j}\right) \boldsymbol{e}_{k}
$$

We will refer to a specific commutator by the index pair $(i, j)$. Acting with the $(1,2)$ commutator on $x$ and $y$, we obtain the

commutator equations

$$
\begin{aligned}
& \boldsymbol{e}_{1} \xi_{2}-\boldsymbol{e}_{2} \xi_{1}=-\alpha_{1} \xi_{1}+\alpha_{2} \xi_{2} \\
& \boldsymbol{e}_{1} v_{2}-\boldsymbol{e}_{2} v_{1}=-\alpha_{1} v_{1}+\alpha_{2} v_{2} .
\end{aligned}
$$

Writing $\boldsymbol{e}_{0}$ and $\boldsymbol{e}_{3}$ as linear combinations of $\partial_{t}$ and $\partial_{\varphi}$, from the $(0,3)$ commutators acting on $x$ and $y$ follows that

$$
\begin{aligned}
& \gamma_{1}=\sigma_{1}+\omega_{1} \\
& \gamma_{2}=\sigma_{2}+\omega_{2}
\end{aligned}
$$

respectively. All the remaining commutator equations are identically satisfied when acting on the essential coordinates $x$ and $y$.

\footnotetext{
${ }^{2}$ During this transformation, the rotation coefficients transform as

$$
\hat{\gamma}_{j k}^{i}=\Lambda_{\ell}^{i} \Lambda_{j}^{m} \Lambda_{k}^{n} \gamma_{m n}^{\ell}+\left(\boldsymbol{e}_{n} \Lambda_{\ell}^{i}\right) \Lambda_{j}^{\ell} \Lambda_{k}{ }^{n},
$$

where $\Lambda_{\ell}^{i}$ is the transformation matrix and $\Lambda_{\ell}^{i} \Lambda_{j}^{\ell}=\delta_{j}^{i}$.
} 
From the relation $R_{b i j}^{a}=e_{i} \gamma_{b j}^{a}-e_{j} \gamma_{b i}^{a}+2 \gamma_{k b[i} \gamma^{a k}{ }_{j]}+2 \gamma_{b k}^{a} \gamma^{k}{ }_{[i j]}$ we obtain the Ricci equations

$$
\begin{aligned}
\boldsymbol{e}_{1} a_{1} & =E_{1}-\frac{1}{6}(\mu+3 p)+a_{1}^{2}+a_{2} \alpha_{1}-\left(\sigma_{2}+\omega_{2}\right)\left(3 \sigma_{2}-\omega_{2}\right) \\
\boldsymbol{e}_{2} a_{2} & =E_{2}-\frac{1}{6}(\mu+3 p)+a_{2}^{2}+a_{1} \alpha_{2}-\left(\sigma_{1}+\omega_{1}\right)\left(3 \sigma_{1}-\omega_{1}\right) \\
\boldsymbol{e}_{2} a_{1} & =E_{3}+a_{1} a_{2}-a_{2} \alpha_{2}+\left(3 \sigma_{1}-\omega_{1}\right)\left(\sigma_{2}+\omega_{2}\right) \\
\boldsymbol{e}_{1} a_{2} & =E_{3}+a_{2} a_{1}-a_{1} \alpha_{1}+\left(3 \sigma_{2}-\omega_{2}\right)\left(\sigma_{1}+\omega_{1}\right) \\
\boldsymbol{e}_{1}\left(\sigma_{1}+\omega_{1}\right) & =-H_{2}-2 a_{2} \omega_{2}+\alpha_{1}\left(\sigma_{2}+\omega_{2}\right)-\beta_{1}\left(\sigma_{1}-\omega_{1}\right)+\beta_{2}\left(\sigma_{2}-\omega_{2}\right) \\
\boldsymbol{e}_{2}\left(\sigma_{2}+\omega_{2}\right) & =-H_{1}-2 a_{1} \omega_{1}+\alpha_{2}\left(\sigma_{1}+\omega_{1}\right)-\beta_{2}\left(\sigma_{2}-\omega_{2}\right)+\beta_{1}\left(\sigma_{1}-\omega_{1}\right) \\
\boldsymbol{e}_{2}\left(\sigma_{1}+\omega_{1}\right) & =H_{3}+2 a_{2} \omega_{1}-\alpha_{2}\left(\sigma_{2}+\omega_{2}\right)-2 \beta_{2} \sigma_{1} \\
\boldsymbol{e}_{1}\left(\sigma_{2}+\omega_{2}\right) & =H_{3}+2 a_{1} \omega_{2}-\alpha_{1}\left(\sigma_{1}+\omega_{1}\right)-2 \beta_{1} \sigma_{2} \\
\boldsymbol{e}_{1} \beta_{1} & =-E_{2}-\frac{1}{3} \mu+\alpha_{1} \beta_{2}-\beta_{1}^{2}-\left(\sigma_{2}-3 \omega_{2}\right)\left(\sigma_{2}+\omega_{2}\right) \\
\boldsymbol{e}_{2} \beta_{2} & =-E_{1}-\frac{1}{3} \mu+\alpha_{2} \beta_{1}-\beta_{2}^{2}-\left(\sigma_{1}-3 \omega_{1}\right)\left(\sigma_{1}+\omega_{1}\right) \\
\boldsymbol{e}_{2} \beta_{1} & =E_{3}-\alpha_{2} \beta_{2}-\beta_{1} \beta_{2}+\left(\sigma_{1}-3 \omega_{1}\right)\left(\sigma_{2}+\omega_{2}\right) \\
\boldsymbol{e}_{1} \beta_{2} & =E_{3}-\alpha_{1} \beta_{1}-\beta_{2} \beta_{1}+\left(\sigma_{2}-3 \omega_{2}\right)\left(\sigma_{1}+\omega_{1}\right)
\end{aligned}
$$

and

$$
\begin{aligned}
\boldsymbol{e}_{1}\left(\sigma_{1}-\omega_{1}\right) & +\boldsymbol{e}_{2}\left(\sigma_{2}-\omega_{2}\right)=-H_{1}-H_{2}+\alpha_{1}\left(\sigma_{2}-\omega_{2}\right)+\alpha_{2}\left(\sigma_{1}-\omega_{1}\right) \\
\boldsymbol{e}_{2} \alpha_{1}+\boldsymbol{e}_{1} \alpha_{2} & =-E_{1}-E_{2}+\frac{1}{3} \mu+\alpha_{1}^{2}+\alpha_{2}^{2} \\
E_{1}+E_{2} & =-\frac{1}{6}(\mu+3 p)-a_{1} \beta_{1}-a_{2} \beta_{2}+\left(\sigma_{1}+\omega_{1}\right)^{2}+\left(\sigma_{2}+\omega_{2}\right)^{2} \\
H_{1}+H_{2} & =-\left(a_{1}+\beta_{1}\right)\left(\sigma_{1}+\omega_{1}\right)-\left(a_{2}+\beta_{2}\right)\left(\sigma_{2}+\omega_{2}\right) .
\end{aligned}
$$

The Bianchi equations $R_{i j[k \ell ; m]}=0$ are not necessary equations (since the space-time is without isotropy [5, 6]), but they may be used as "help" equations (although all information in them are contained in the lower-derivative stage of the Ricci equations for stationary axisymmetric space-times). Thus we have the twice contracted Bianchi equations $T^{i j}{ }_{j}=0$

$$
\begin{aligned}
& \boldsymbol{e}_{1} p=a_{1}(\mu+p) \\
& \boldsymbol{e}_{2} p=a_{2}(\mu+p),
\end{aligned}
$$

and the

Bianchi equations

$$
\begin{aligned}
\boldsymbol{e}_{1}\left(E_{1}+E_{2}\right) & =\left(a_{1}-2 \beta_{1}\right) E_{1}+\left(2 a_{1}-\beta_{1}\right) E_{2}-\left(a_{2}+\beta_{2}\right) E_{3} \\
& +\left(\sigma_{1}+\omega_{1}\right)\left(H_{1}-H_{2}\right)+2\left(\sigma_{2}+\omega_{2}\right) H_{3}-\frac{1}{6} e_{1} \mu \\
\boldsymbol{e}_{2}\left(E_{2}+E_{1}\right) & =\left(a_{2}-2 \beta_{2}\right) E_{2}+\left(2 a_{2}-\beta_{2}\right) E_{1}-\left(a_{1}+\beta_{1}\right) E_{3} \\
& +\left(\sigma_{2}+\omega_{2}\right)\left(H_{2}-H_{1}\right)+2\left(\sigma_{1}+\omega_{1}\right) H_{3}-\frac{1}{6} e_{2} \mu \\
\boldsymbol{e}_{1} E_{1}+e_{2} E_{3} & =\left(\alpha_{2}-2 \beta_{1}\right) E_{1}-\left(\alpha_{2}+\beta_{1}\right) E_{2}+\left(2 \alpha_{1}-\beta_{2}\right) E_{3} \\
& +\left(\sigma_{1}+3 \omega_{1}\right) H_{1}+2 \sigma_{1} H_{2}-\left(\sigma_{2}-3 \omega_{2}\right) H_{3}-\frac{1}{3} e_{1} \mu \\
\boldsymbol{e}_{2} E_{2}+e_{1} E_{3} & =\left(\alpha_{1}-2 \beta_{2}\right) E_{2}-\left(\alpha_{1}+\beta_{2}\right) E_{1}+\left(2 \alpha_{2}-\beta_{1}\right) E_{3} \\
& +\left(\sigma_{2}+3 \omega_{2}\right) H_{2}+2 \sigma_{2} H_{1}-\left(\sigma_{1}-3 \omega_{1}\right) H_{3}-\frac{1}{3} e_{2} \mu
\end{aligned}
$$


and

$$
\begin{aligned}
\boldsymbol{e}_{1}\left(H_{1}+H_{2}\right) & =\left(a_{1}-2 \beta_{1}\right) H_{1}+\left(2 a_{1}-\beta_{1}\right) H_{2}-\left(a_{2}+\beta_{2}\right) H_{3} \\
& +\left(\sigma_{1}+\omega_{1}\right)\left(E_{2}-E_{1}\right)-2\left(\sigma_{2}+\omega_{2}\right) E_{3}+\frac{1}{2}\left(\sigma_{1}+\omega_{1}\right)(\mu+p) \\
\boldsymbol{e}_{2}\left(H_{2}+H_{1}\right) & =\left(a_{2}-2 \beta_{2}\right) H_{2}+\left(2 a_{2}-\beta_{2}\right) H_{1}-\left(a_{1}+\beta_{1}\right) H_{3} \\
& +\left(\sigma_{2}+\omega_{2}\right)\left(E_{1}-E_{2}\right)-2\left(\sigma_{1}+\omega_{1}\right) E_{3}+\frac{1}{2}\left(\sigma_{2}+\omega_{2}\right)(\mu+p) \\
\boldsymbol{e}_{1} H_{1}+e_{2} H_{3} & =\left(\alpha_{2}-2 \beta_{1}\right) H_{1}-\left(\alpha_{2}+\beta_{1}\right) H_{2}+\left(2 \alpha_{1}-\beta_{2}\right) H_{3} \\
& -\left(\sigma_{1}+3 \omega_{1}\right) E_{1}-2 \sigma_{1} E_{2}+\left(\sigma_{2}-3 \omega_{2}\right) E_{3}+\omega_{1}(\mu+p) \\
\boldsymbol{e}_{2} H_{2}+e_{1} H_{3} & =\left(\alpha_{1}-2 \beta_{2}\right) H_{2}-\left(\alpha_{1}+\beta_{2}\right) H_{1}+\left(2 \alpha_{2}-\beta_{1}\right) H_{3} \\
& -\left(\sigma_{2}+3 \omega_{2}\right) E_{2}-2 \sigma_{2} E_{1}+\left(\sigma_{1}-3 \omega_{1}\right) E_{3}+\omega_{2}(\mu+p) .
\end{aligned}
$$

Once more, we stress the fact that in order to find a solution to Einstein's equations, we need to solve Eqs. (16), (17), and (20)-(35). The use of the Bianchi equations (38)-(45) is optional when no further symmetry restrictions are imposed.

\section{Magnetic space-times}

For space-times with a purely magnetic Weyl tensor, we set $E_{A}=0$.

\subsection{Incompressible fluids}

Incompressible fluids are defined by $\boldsymbol{e}_{1} \mu=\boldsymbol{e}_{2} \mu=0$. We use the rotation (12) to set $H_{3}=0$. We may then prove the following:

Theorem 1. The only incompressible axistationary perfect fluid with a pure magnetic Weyl tensor is the interior Schwarzschild solution.

Proof. From Eqs. (38)-(41) we get four algebraic equations from which $H_{1}=H_{2}$ and $\sigma_{1}+\omega_{1}=\sigma_{2}+\omega_{2}=0$. But Eq. (35) then results in $H_{1}=H_{2}=0$, i.e., the space-time is conformally flat. According to a theorem by Collinson [7], the only conformally flat axistationary perfect fluid space-time is the interior Schwarzschild solution.

\subsection{Rigidly rotating fluids}

When the fluid is rigid, we have $\sigma_{1}=\sigma_{2}=0$. We set $\omega_{1}=0$ by using the transformation (12). Then the Bianchi equations (38)-(41) give the constraints

$$
\omega_{2} H_{3}=0, \quad \omega_{2}\left(2 H_{1}+H_{2}\right)=0 .
$$

If $\omega_{2}=0$, the fluid is static and Eqs. (38) and (39) imply $\boldsymbol{e}_{1} \mu=\boldsymbol{e}_{2} \mu=0$, while Eqs. (24), (26), and (25) gives $H_{1}=H_{2}=H_{3}=0$. Thus, this is the interior Schwarzschild solution. 
If $H_{3}=0$ and $H_{2}=-2 H_{1}$, we obtain a consistent subsystem of equations

$$
\begin{aligned}
& \boldsymbol{e}_{1} p=\boldsymbol{e}_{1} \mu=\boldsymbol{e}_{1} \beta_{2}=\boldsymbol{e}_{1} a_{2}=0 \\
& \boldsymbol{e}_{2} a_{2}=a_{2}^{2}-\frac{1}{6}(\mu+3 p) \\
& \boldsymbol{e}_{2} \beta_{2}=-\frac{1}{3} \mu-\beta_{2}^{2} \\
& \boldsymbol{e}_{2} \mu=-3\left(a_{2}+\beta_{2}\right)\left(\mu+3 p+6 a_{2} \beta_{2}\right) \\
& \boldsymbol{e}_{2} p=a_{2}(\mu+p) \\
& \boldsymbol{e}_{1} \beta_{1}=-b^{2}-\beta_{1}^{2} \\
& \boldsymbol{e}_{2} \beta_{1}=-\beta_{1} \beta_{2},
\end{aligned}
$$

where $b^{2} \equiv \beta_{2}^{2}-3 a_{2} \beta_{2}-\frac{1}{6} \mu-\frac{3}{2} p$. (For the interior Schwarzschild solution $b^{2}>0$.) This system is characterized by $\omega_{1}=a_{1}=\alpha_{2}=0, \alpha_{1}=-\beta_{2}, H_{1}=\left(a_{2}+\beta_{2}\right) \omega_{2}$, and $\omega_{2}^{2}=(\mu+3 p) / 6+a_{2} \beta_{2}$. We choose coordinates $\{x, y\}$ such that $\boldsymbol{e}_{1}=\zeta(\partial / \partial x)$, where we assume that $\zeta \equiv \zeta(y)$ depends only on $y$, and $\boldsymbol{e}_{2}=\xi(\partial / \partial x)+v(\partial / \partial y)$. With this choice of coordinates, all quantities except $\beta_{1}$ become independent of $x$. We still have the freedom to make the coordinate transformations

$$
\begin{aligned}
& \bar{x}=h_{1}(y) x+h_{0}(y) \\
& \bar{y}=\bar{y}(y),
\end{aligned}
$$

where $h_{1}(y)$ and $h_{0}(y)$ are some $x$-independent functions. It follows from the $(1,2)$ commutator on $y$ (cf. Eq. (17)), that $v$ depends only on $y$. Acting with the commutator $(1,2)$ on $x$ (cf. Eq. (16)), we find that

$$
\zeta \frac{\partial \xi}{\partial x}-v \frac{\partial \zeta}{\partial y}=\zeta \beta_{2}
$$

Integrating, we obtain

$$
\xi=\left(\beta_{2}+\frac{v}{\zeta} \frac{\partial \zeta}{\partial y}\right) x+\xi_{0}
$$

where $\xi_{0}$ is a function depending only on the coordinate $y$. Performing a transformation (54), $\xi$ becomes

$$
\bar{\xi}=\xi h_{1}(y)+v\left(x \frac{d h_{1}(y)}{d y}+\frac{d h_{0}(y)}{d y}\right) .
$$

Substituting (57), we can achieve $\bar{\xi}=0$ by suitably choosing $h_{1}(y)$ and $h_{0}(y)$. This means that we can use a coordinate system in which

$$
\boldsymbol{e}_{1}=\zeta \frac{\partial}{\partial x}, \quad \boldsymbol{e}_{2}=v \frac{\partial}{\partial y},
$$

and both $\zeta$ and $v$ depend only on $y$. Since $b$ does not depend on $x$ either, we can integrate (52) to obtain

$$
\beta_{1}=-b \tan \left[\frac{b}{\zeta}\left(x-x_{0}\right)\right],
$$

where $x_{0}$ is some function of $y$. Substituting $\xi$ and $\beta_{1}$ in Eq. (53) and using $\boldsymbol{e}_{2} b=-\beta_{2} b$ we get that $x_{0}$ must be a constant. Since we only used the derivative of $h_{0}(y)$ in the transformation (58), we still may add a constant to the coordinate $x$, and we use this freedom to set $x_{0}=0$. 
The remaining equations are

$$
\begin{aligned}
& v \frac{d a_{2}}{d y}=a_{2}^{2}-\frac{1}{6}(\mu+3 p) \\
& v \frac{d \beta_{2}}{d y}=-\frac{1}{3} \mu-\beta_{2}^{2} \\
& v \frac{d \mu}{d y}=-3\left(a_{2}+\beta_{2}\right)\left(\mu+3 p+6 a_{2} \beta_{2}\right) \\
& v \frac{d p}{d y}=a_{2}(\mu+p) .
\end{aligned}
$$

By introducing $B \equiv b^{2}, \Omega \equiv \omega_{2}^{2}$, these equations can also be written as

$$
\begin{aligned}
& v \frac{d B}{d y}=-2 B \beta_{2} \\
& v \frac{d \beta_{2}}{d y}=B-3 \Omega \\
& v \frac{d a_{2}}{d y}=a_{2}\left(a_{2}+\beta_{2}\right)-\Omega \\
& v \frac{d \Omega}{d y}=-2 \Omega\left(a_{2}+2 \beta_{2}\right)
\end{aligned}
$$

The equation of state $\mu=\mu(p)$ is given by a lengthy third-order nonlinear differential equation. $\mu=$ constant is the interior Schwarzschild solution (see Theorem 1).

We see that in the static limit $\omega_{2} \rightarrow 0$, we obtain $H_{1}=H_{2}=0$ and $\boldsymbol{e}_{1} \mu=\boldsymbol{e}_{2} \mu=0$. Thus in this limit we obtain the interior Schwarzschild solution. The acceleration $a_{2}$ is expressible in terms of $p$ :

$$
a_{2}^{2}=\frac{2}{3} \mu+p+D(\mu+p)^{2}
$$

where $D$ is a constant. For spherical symmetry, the quantities in the set $S$ are invariant under the action of a 3 -dimensional isotropy group at the center of the spheres. Thus, denoting center values by an index $c,\left(a_{2}\right)_{c}=0,[$ and

$$
D=-\frac{1}{3} \frac{2 \mu_{c}+3 p_{c}}{\left(\mu_{c}+p_{c}\right)^{2}}
$$

We collect our results in the following theorem:

Theorem 2. The only rigidly rotating axistationary perfect fluid solutions with a pure magnetic Weyl tensor are the interior Schwarzschild solution and the space-time given by Eqs. (61)-64).

\section{Restoring the metric}

We can always generate the set $S$ from the metric (11) in the boosted frame (7). Thus, to obtain the full metric we only need to apply the commutators to the two coordinates $t$ and

\footnotetext{
${ }^{3}$ This follows since $a_{2}$ is the 2-component of the acceleration covector which must be zero at the center, otherwise there is a preferred direction.
} 
$\varphi$ of which all geometric quantities are independent, since the 1 -forms $\boldsymbol{\omega}^{1}$ and $\boldsymbol{\omega}^{2}$ are known from solving the necessary and sufficient equations. Thus, to obtain the "missing part" of the metric, we must integrate

$$
\begin{aligned}
& {\left[\boldsymbol{e}_{i}, \boldsymbol{e}_{j}\right] t=\left(\gamma^{0}{ }_{j i}-\gamma^{0}{ }_{i j}\right) \boldsymbol{e}_{0} t+\left(\gamma^{3}{ }_{j i}-\gamma^{3}{ }_{i j}\right) \boldsymbol{e}_{3} t} \\
& {\left[\boldsymbol{e}_{i}, \boldsymbol{e}_{j}\right] \boldsymbol{\varphi}=\left(\gamma^{0}{ }_{j i}-\gamma^{0}{ }_{i j}\right) \boldsymbol{e}_{0} \varphi+\left(\gamma^{3}{ }_{j i}-\gamma^{3}{ }_{i j}\right) \boldsymbol{e}_{3} \varphi}
\end{aligned}
$$

where the form of the right hand side follows from the fact that $\boldsymbol{e}_{1}$ and $\boldsymbol{e}_{2}$ are linear combinations of $\partial_{x}$ and $\partial_{y}$. Because of the complicated form of $\boldsymbol{e}_{i}$, the commutator equations (71) and (72) will in general be difficult to integrate. This is of no physical importance, though, since we know that the set $S$ gives a complete local description of the space-time. The central dilemma is the condition for matching a given fluid solution to a vacuum counterpart. This problem can in principle be solved using the metric description, while no such procedure exists (yet) for the description in terms of $S$.

\subsection{The shear-free case}

When the fluid is shear-free, i.e., rigidly rotating, we may put the boost parameter $\chi$ equal to zero, since the frame (2) is comoving in the rigid case. The expressions (10) and (11) for $\boldsymbol{e}_{0}$ and $\boldsymbol{e}_{3}$, respectively, simplify considerably:

$$
\begin{aligned}
\boldsymbol{e}_{0} & =f^{-1 / 2} \partial_{t} \\
\boldsymbol{e}_{3} & =\frac{f^{1 / 2}}{\varrho}\left(\partial_{\varphi}-\omega \partial_{t}\right) .
\end{aligned}
$$

Here $f, \varrho$ and $\omega$ are functions of $x$ and $y$.

We proceed to determining the $x$ (angular) dependence of the metric quantities by use of the commutator equations $(71)$ and $(72)$. From the $(0,1)$ commutator acting on $t$, we get $\partial f / \partial x=0$, and hence $f$ is a function of $y$ only. Integrating the equation obtained from the $(1,3)$ commutator acting on $\varphi$, we get

$$
\varrho=\varrho_{0} \cos \left(\frac{b}{\zeta} x\right),
$$

while acting on $t$ gives

$$
\omega=-\frac{2}{b f} \omega_{2} \varrho_{0} \sin \left(\frac{b}{\zeta} x\right)+\omega_{0},
$$

where $\varrho_{0}$ and $\omega_{0}$ depend on $y$ only. The commutator equation $(0,2)$ acting on $t$ and $(1,2)$ acting on $x$ give, respectively

$$
\begin{aligned}
& a_{2}=-\frac{v}{2} \frac{d}{d y} \ln f \\
& \beta_{2}=-v \frac{d}{d y} \ln \zeta .
\end{aligned}
$$

Comparison with (65) shows $b=C \zeta$ where one can set the constant $C=1$ by rescaling the coordinate $x$. The action of $(2,3)$ on $\varphi$ yields

$$
\zeta=C^{\prime} \frac{\sqrt{f}}{\rho_{0}}
$$


where we can arrange the constant $C^{\prime}=1$ by rescaling the coordinate $\varphi$.

Substituting $\beta_{2}$ in the $(2,3)$ commutator acting on $t$, the resulting equation can be separated into $x$-dependent and $x$-independent terms. From the $x$-independent part it follows that $\omega_{0}$ is a constant. Hence we can set $\omega_{0}=0$ by $t \rightarrow t+\omega_{0} \varphi$. The terms proportional to $\sin (b x)$ can be integrated,

$$
2 \omega_{2}=K \sqrt{f} \zeta^{2}
$$

where $K$ is a constant. Putting together these results, we find $\omega=K \sin x$. By use of the transformation (55), we arrange $v=\sqrt{f}$. The space-time metric takes the form ( $c f$. Eq. (1D)

$$
\mathrm{d} s^{2}=f(\mathrm{~d} t+K \sin x \mathrm{~d} \varphi)^{2}-\frac{1}{f}\left[\mathrm{~d} y^{2}+\rho_{0}^{2}\left(\mathrm{~d} x^{2}+\cos ^{2} x \mathrm{~d} \varphi^{2}\right)\right] .
$$

The angular dependence of this metric is identical with the angular dependence of the metric in Ref. [8]. In that reference, the Ansatz is used that the eigenrays of the Killing vector $\partial / \partial t$ are geodesic. Both solutions are of NUT type. For a full understanding of the relationship of the physical assumptions used in both papers, a study of the radial behavior is needed.

\section{$5 \quad$ Petrov types}

In this section, we classify the circularly rotating perfect fluid space-times into Petrov types. A Newman-Penrose null tetrad convenient for this investigation is given by

$$
\begin{aligned}
& \mathbf{l}=\frac{1}{\sqrt{2}}\left(\boldsymbol{e}_{0}+\boldsymbol{e}_{3}\right) \\
& \mathbf{n}=\frac{1}{\sqrt{2}}\left(\boldsymbol{e}_{0}-\boldsymbol{e}_{3}\right) \\
& \mathbf{m}=\frac{1}{\sqrt{2}}\left(\boldsymbol{e}_{1}+i \boldsymbol{e}_{2}\right) .
\end{aligned}
$$

The nonvanishing Weyl spinor components are

$$
\begin{aligned}
\Psi_{0} & =\frac{1}{2}\left[E_{2}-E_{1}-2 H_{3}+i\left(H_{1}-H_{2}-2 E_{3}\right)\right] \\
\Psi_{2} & =\frac{1}{2}\left[E_{1}+E_{2}-i\left(H_{1}+H_{2}\right)\right] \\
\Psi_{4} & =\frac{1}{2}\left[E_{2}-E_{1}+2 H_{3}+i\left(H_{1}-H_{2}+2 E_{3}\right)\right] .
\end{aligned}
$$

The algebraic Petrov type is determined by the roots of the equation

$$
\Psi_{4} b^{4}+6 \Psi_{2} b^{2}+\Psi_{0}=0 .
$$

The Petrov type is II if and only if either $\Psi_{0}=0$ or $\Psi_{4}=0$. (Reversal of the $\boldsymbol{e}_{3}$ direction interchanges $\Psi_{0}$ and $\Psi_{4}$.) Petrov D type fields are characterized by the conditions

$$
\begin{aligned}
& 4 E_{1} H_{1}+5 E_{1} H_{2}+5 E_{2} H_{1}+4 E_{2} H_{2}-2 E_{3} H_{3}=0 \\
& 2 E_{1}^{2}+5 E_{1} E_{2}+2 E_{2}^{2}-E_{3}^{2}-2 H_{1}^{2}-5 H_{1} H_{2}-2 H_{2}^{2}+H_{3}^{2}=0 .
\end{aligned}
$$

The type is $\mathrm{N}$ if $\Psi_{0}=\Psi_{2}=0$. Type III is excluded by equation (88). 
From the point of view of star modeling, type N perfect fluids can hardly be of any significance. This is due to the following

Theorem 3. A type $N$ axistationary perfect fluid spacetime has a constant pressure $p$ and density $\mu$.

Proof. The equations $\Psi_{0}=\Psi_{2}=0$, with (85) and (86) yield $E_{2}=H_{3}=-E_{1}$ and $E_{3}=-H_{2}=H_{1}$. We next arrange $\omega_{2}+\sigma_{2}=0$ by using the transformation (12). We obtain $H_{1}$ and $E_{1}$ from the algebraic equations (25) and (27), respectively. The equation of state $\mu+p=0$ follows at once from (30). From the Bianchi identities (36) and (37) the statement of the theorem follows.

\subsection{Rigidly rotating electric space-times}

In this subsection we consider rigidly rotating fluid spacetimes with purely electric Weyl tensor. We then have $\sigma_{1}=\sigma_{2}=0$ and $H_{A}=0$. These spacetimes have the property stated in the following

Theorem 4. A rigidly rotating purely electric perfect fluid space-time is of Petrov type D. The four-velocity lies in the plane spanned by the principal null directions of the Weyl tensor.

Proof. We set $\omega_{1}=0$ by using the transformation (12). From the Bianchi equations (42), (44) and (45) we get, respectively

$$
\begin{aligned}
& E_{3}=0 \\
& E_{2}=-2 E_{1} \\
& \mu=3 E_{2}+p .
\end{aligned}
$$

The combination of Bianchi equations (38)-(41) yields

$$
\begin{aligned}
& \alpha_{2}=0 \\
& \beta_{2}=-\alpha_{1} .
\end{aligned}
$$

From the Ricci equations (35) and (34) we have, respectively

$$
\begin{aligned}
& \alpha_{1}=a_{2} \\
& E_{1}=\frac{1}{2}\left(a_{1} \beta_{1}-a_{2}^{2}-\omega_{2}^{2}\right)+\frac{1}{6} p .
\end{aligned}
$$

From conditions (89) and (90) it follows immediately that these fields are of type D. The double principal null directions are $\mathbf{l}+\mathbf{n}+i(\mathbf{m}-\overline{\mathbf{m}})$ and $\mathbf{l}+\mathbf{n}-i(\mathbf{m}-\overline{\mathbf{m}})$. Hence the fourvelocity $\boldsymbol{e}_{0}$ lies in the plane of the principal null directions.

The condition that the rigidly rotating perfect fluid space-time is Petrov type D and that the four-velocity is a linear combination of the principal directions has been thoroughly investigated by Senovilla 9, who gave all the exact solutions in this class.

\section{Acknowledgment}

This work has been supported by OTKA fund T022533, the Royal Swedish Academy of Sciences, and the Hungarian Academy of Sciences. Thanks are due to C. McIntosh for calling our attention to magnetic fluids. 


\section{References}

[1] De Felice, F. and C. J. S. Clarke (1990) Relativity on curved manifolds, pp. 254-255 (Cambridge: Cambridge University Press).

[2] Arianrhod et al. (1994) Magnetic curvatures, Class. Quantum Grav. 11, 2331-2335.

[3] Misra et al. (1968) A solution of the field equations representing a gravitational field of a magnetic type, Tensor 19, 203-205.

[4] McIntosh et al. (1994) Electric and magnetic Weyl tensors: Classification and analysis, Class. Quantum Grav.11, 1555.

[5] Bradley, M. and A. Karlhede (1991) On the curvature description of gravitational fields, Class. Quantum Grav. 7 449-463.

[6] Bradley, M. and M. Marklund (1996) Finding solutions to Einstein's equations in terms of invariant objects, Class. Quantum Grav. 13 3021-3037.

[7] Collinson, C. D. (1976) The Uniqueness of the Schwarzschild Interior Metric, Gen. Rel. Grav. 7 419-422.

[8] Lukács, B. et al. (1983) A NUT-like solution with fluid matter, Gen. Rel. Grav. 15 $567-571$.

[9] Senovilla, J.M.M. (1987) On Petrov type-D stationary axisymmetric rigidly rotating perfect fluid metrics, Class. Quantum Grav. 4 L115-L119. 